Ann. Génét. Sél. anim., I973, 5 (4), 435-440.

\title{
NOUVELLES OBSERVATIONS SUR UNE FUSION CENTRIQUE CHEZ BOS TAURUS L.
}

\author{
C.-P. POPESCU \\ avec la collaboration technique de Jeaninne Boscher \\ U. N.C. E. I. A. et Laboratoire de Génétique factorielle, \\ Centre national de Recherches zootechniques, I. N. R. A., \\ 78350 Jouy en Josas
}

\section{RÉSUMMÉ}

Une étude cytogénétique a été entreprise sur 220 animaux dont 749 9ㅇ descendantes de $3 \sigma^{\star} \sigma^{*}$ porteurs d'une fusion centrique et 146 o $\delta$ et o $q$ présentant des troubles de fertilité. Dans le premier groupe un rapport de ségrégation I : I est évident. Dans le deuxième groupe, 7 cas nouveaux de fusion centrique ont été identifiés, dont un à l'état homozygote. Certains aspects concernant l'origine et les effets de cette anomalie sont discutés.

\section{INTRODUCTION}

Il a été démontré que certaines anomalies chromosomiques structurales interfèrent chez les bovins avec des troubles de fertilité. Ainsi, une fusion de type robertsonien, impliquant le plus grand et, probablement, le plus petit autosome, anomalie très répandue dans cette espèce, entraîne une baisse de fertilité et un taux d'élimination élevé chez les femelles porteuses (Gustavsson, I97I). Un autre type de translocation appelée " en tandem " provoque, elle aussi, une baisse de fertilité d'environ Io p. Ioo chez les mâles et diminue légèrement la fertilité des femelles (HANSEN, I969).

Les risques considérables de propagation d'une anomalie chromosomique par l'insémination artificielle, presque généralisée actuellement chez les bovins, incitent à rechercher la présence éventuelle de ces anomalies dans toutes les races bovines, leur mode de transmission et leurs effets. 
Le mémoire contient les résultats d'une étude cytogénétique entreprise sur des animaux appartenant à plusieurs races françaises, à la suite de la mise en évidence de deux cas de fusion centrique de type I/29 (POPESCU, I97I).

\section{MATÉRIEL ET MÉTHODES}

L'étude porte sur 220 animaux dont la répartition par race est donnée dans le tableau $\mathbf{r}$. Soixante quatorze animaux étudiés étaient des femelles descendantes de 3 mâles porteurs d'une fusion centrique, dont 2 hétérozygotes et un homozygote pour cette fusion et I 46 des mâles présentant des troubles de fertilité et des femelles appartenant à un lot expérimental dans lequel la présence de cette anomalie avait été signalée.

TABLEAU I

Répartition selon la race des animaux étudiés

\begin{tabular}{|c|c|}
\hline Race & $\begin{array}{c}\text { Nombre d'animaux } \\
\text { étudiés }\end{array}$ \\
\hline Charolaise $\ldots \ldots \ldots \ldots \ldots \ldots \ldots \ldots$ & 66 \\
\hline 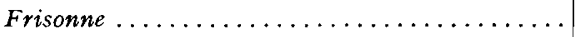 & 10 \\
\hline Montbéliarde $\quad \ldots \ldots \ldots \ldots \ldots \ldots \ldots$ & 82 \\
\hline Normande $\ldots \ldots \ldots \ldots \ldots \ldots \ldots \ldots$ & 28 \\
\hline Limousine $\ldots \ldots \ldots \ldots \ldots \ldots \ldots \ldots$ & 4 \\
\hline Pie rouge de l'Est $\ldots \ldots \ldots \ldots \ldots \ldots \ldots$ & 2 \\
\hline 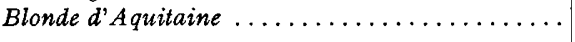 & 28 \\
\hline Total $\ldots \ldots \ldots \ldots \ldots \ldots \ldots \ldots \ldots \ldots \ldots \ldots \ldots$ & 220 \\
\hline
\end{tabular}

L'examen chromosomique a été fait sur des préparations obtenues à partir du sang intégral, selon la méthode de Grouchy et al. (1964). Pour chaque animal, le plus grand nombre possible de métaphases a été étudié et les meilleurs photographiés avec un microscope Leitz-Ortholux sur un film Kodak Microfile. Pour chaque animal trouvé porteur d'une anomalie, plusieurs caryo. types ont été établis et les cultures ont été souvent répétées une ou deux fois.

\section{RÉSULTATS}

a) Les 74 femelles descendantes des 3 mâles porteurs d'une fusion centrique mises à part, nous avons trouvé 7 cas de fusion centrique (tabl. 2) sur I 46 animaux étudiés. On remarque la présence de 6 cas de fusion centrique à l'état hétérozygote et d'un cas homozygote (fig. I $a$ et $b$ ). Les deux premiers cas ont fait l'objet d'une note préliminaire (POPESCU, I97I).

b) En ce qui concerne la descendance des deux mâles hétérozygotes pour la fusion centrique (tabl. 3 , cas I et 2 ) on trouve une proportion à peu près égale d'animaux normaux et d'animaux hétérozygotes avec, toutefois, une légère prédominance 


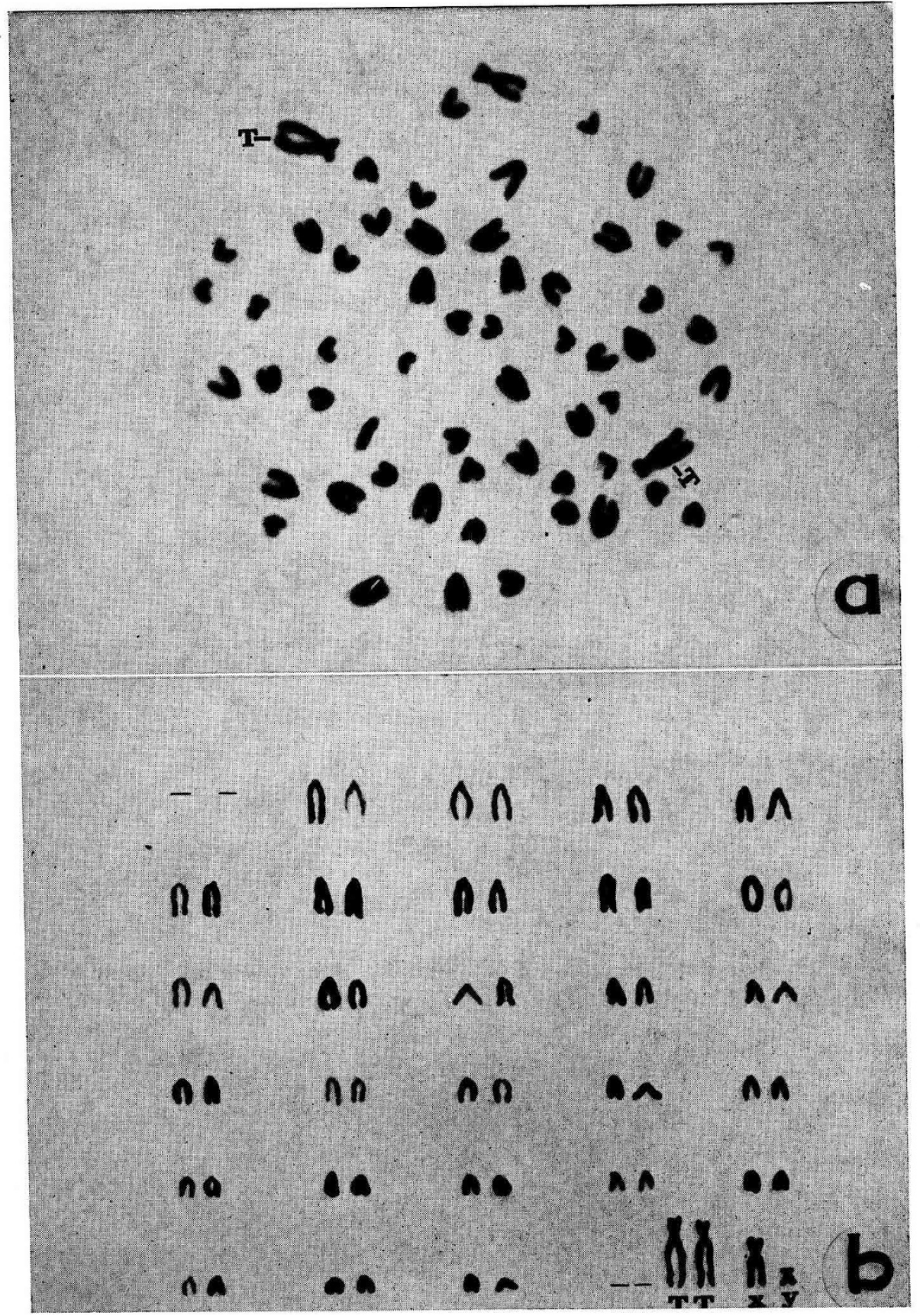

FrG. I. - a) Métaphase présentant une fusion centrique à l'état homozygote ( $\mathrm{T}$ : chromosome transloqué)

b) Caryotype d'une cellule présentant la fusion centrique à l'état homozygote 
TABI,EAU 2

Les cas d'anomalies chromosomiques relevés sur 146 animaux étudiés

\begin{tabular}{l|l}
\hline \hline \\
Cas
\end{tabular}

$\mathrm{T}^{*}=$ Chromosome transloqué.

des hétérozygotes dans le premier cas. Les 20 descendantes du troisième mâle, homozygote pour la même fusion, sont toutes hétérozygotes. On peut encore noter l'absence totale dans la descendance des trois animaux d'individus homozygotes.

\section{TABI,EAU 3}

La transmission de la fusion centrique dans la descendance des trois mâles porteurs

\begin{tabular}{|c|c|c|c|c|c|c|}
\hline \multirow[b]{2}{*}{ Cas } & \multirow[b]{2}{*}{ Race } & \multirow[b]{2}{*}{ Caryotype } & \multicolumn{3}{|c|}{ Descendants } & \multirow[b]{2}{*}{ Total } \\
\hline & & & $\begin{array}{l}\text { Caryotype normal } \\
\qquad(2 \mathrm{n}=60)\end{array}$ & $\begin{array}{c}\text { Caryotype } \\
2 \mathrm{n}=59+\mathrm{T}\end{array}$ & $\begin{array}{c}\text { Caryotype } \\
2 \mathrm{n}=58+\mathrm{TT}\end{array}$ & \\
\hline 1 & Montbéliarde & $2 \mathrm{n}=59+\mathrm{T}$ & 17 & 22 & 一 & 39 \\
\hline 2 & Montbéliarde & $2 \mathrm{n}=59+\mathrm{T}$ & 7 & 8 & $一$ & 15 \\
\hline 3 & Charolaise & $2 \mathrm{n}=58+\mathrm{TT}$ & - & 20 & 一 & 20 \\
\hline
\end{tabular}

\section{DISCUSSION}

La fusion centrique de type $\mathrm{r} / 29$ a été identifiée dans une dizaine de races bovines réparties dans plusieurs pays d'Europe et d'Amérique (Gustavsson, I966, I969; HERSCHLER et FECHHEIMER, I966; RUGIATI et FEDRIGo, I967 ; RIECK et al., I968 ; AMRUD, I969 ; HARVEY, I97I, I972 ; POPESCU, I97I ; DARRÉ et al., I972 ; FORGET et al., I972). Deux hypothèses pourraient être invoquées pour expliquer sa présence dans un nombre de races relativement important et sa large distribution géogra- 
phique : mutation récurrente ou origine commune. Actuellement il n'existe pas suffisamment d'arguments pour retenir définitivement l'une ou l'autre de ces deux possibilités. Toutefois, certains auteurs trouvent plus plausible 1'hypothèse d'une origine commune de cette mutation, apparue chez un ancêtre des races actuelles (Gustavsson, I969; Popescu, I97I). Plusieurs constatations plaideraient en sa faveur. Ainsi, dans aucun système de polymorphisme chromosomique comportant une translocation de ce type, l'existence d'une mutation récurrente n'a été prouvée. Par contre, dans deux cas de fusion centrique, ayant une grande distribution géographique, l'origine commune de l'anomalie a été démontrée (STAIGER, I954; Smrth, I962, cités par Gustavsson, I969).

Dans des études faites sur des lignées cellulaires bovines, normales ou transformées (NACHTIGAL et al., I970), rien n'indique une tendance particulière des chromosomes $I$ et 29 à fusionner.

Sur le plan plus général Hsu et MEAD (I969) affirment que la probabilité pour que deux remaniements chromosomiques identiques se produisent est très faible et TAYLOR et al. (I969) considèrent que chaque translocation de type robertsonien est un événement unique. D'autre part, il est admis que les bivalents ont une disposition orientée pendant les premiers stades de la prophase méiotique. Certains d'entre eux sont attachés au nucléole et contiennent les organisateurs nucléolaires. Ce sont justement ceux-ci qui, du fait de leur proximité, sont les plus susceptibles de subir des remaniements, tels les translocations (FERGUSON-SMrTh, I97I). Chez Bos taurus, les difficultés d'identification des chromosomes, à cause de leur morphologie semblable n'ont pas permis de préciser la disposition des chromosomes impliqués dans la fusion, durant la prophase méiotique. La participation des chromosomes I et 29 en tant qu'organisateurs nucléolaires pourrait être invoquée en faveur d'une mutation récurrente.

Quant au mode de transmission de cette anomalie il est évident (tabl. 3) qu'il se fait en proportions égales I : I, ce qui confirme les observations antérieures (GusTAvsson, I969). Chez d'autres espèces on a observé un avantage net des hétérozygotes équilibrés par rapport aux descendants normaux (HAMERTON, I968, I969 ; POPESCU, I972) dû probablement à une sélection prézygotique. La même constatation faite dans le cas de l'animal I (tabl. 3) ne peut pas être prise en considération en raison de 1'effectif réduit analysé. Une conséquence certaine de cette anomalie est la fertilité réduite des femelles porteuses (Gustavsson, I969) qui s'explique par la formation des gamètes non-équilibrés à la suite d'une mauvaise ségrégation. Les zygotes issus des fécondations avec des gamètes disomiques ou multisomiques pour l'une ou l'autre des chromosomes fusionnés, seraient léthaux et augmenteraient la mortalité embryonnaire.

Sur le plan génétique il est aussi possible que la fusion centrique diminue le nombre de chiasmas et entraîne un effet de position. Par ailleurs, il semble que la formation de la fusion centrique entraîne la perte d'un centromère, puisque le chromosome fusionné traité par des techniques appropriées, montre un seul bloc d'hétérochromatine constitutive (Popescu, I973). Seule 1'inactivité génétique de 1'hétérochromatine constitutive pourrait expliquer l'absence de conséquences de cette perte pour l'animal porteur. 


\section{SUMMARY}

\section{NEW OBSERVATIONS ON CENTRIC FUSION IN BOS TAURUS L.}

A cytogenetic analysis of 220 animals was performed : 74 females offspring of 3 males, carriers of centric fusion, and $146 \delta \delta^{\star}$ and $q$ 우 with reduced fertility. In the first group, the segregation ratio appears to be I : I. In the second group, 7 new carriers of centric fusion were found. Some aspects of the origin and implications of this abnormality are discussed.

\section{RÉFÉRENCES BIBLIOGRAPHIQUES}

Amrud J., 1969. Centric fusion of chromosomes in norvegian red cattle (N. R. F.). Hereditas, 62, 293-302.

Darré R., Queinnec G., Berland H. M., 1972. La translocation I-29 des bovins. Étude générale et importance du phénomène dans le Sud-Ouest. Revue Méd. Vét., 123, 477-494.

Ferguson-Smith M. A., I972. Human chromosomes in meiosis. In de Grouchy et al. Proceeding of the Fourth International Congress of Human Genetics. Paris, september, I97I. Excerpta Medica, Amsterdam, I95-2II.

Froget J., Coulon J., Nain M. C., Daubiez J. M., x972. Anomalie chromosomique de type fusion centrique chez un veau Charolais. Bull. Soc. Sci. Vét. et Méd. comparée (Lyon), 74, I3I-I35.

Grouchy J. de, Roubin M., Passage E., I964. Microtechnique pour l'étude des chromosomes humains à partir d'une culture de leucocytes sanguins. Ann. Génét., \%, 45 .

Gustavsson I., 1966. Chromosome abnormality in cattle. Nature, 211, 865-866.

Gustavsson I., 1969. Cytogenetics distribution and phenotypic effects of a translocation in Swedish cattle. Hereditas, 63, 68-I69.

HAMERTON J. L., I 968 . Robertsonian translocations in man : evidence for prezygotic selection. Cytogenetics, $7,260-276$.

Hamerton J. L., x 969 . Reciprocal translocations in man. In C. D. Darlington and K. R. Lewis. Chromosomes today, 11, $2 \mathrm{I}-32$.

HANSEN K. M., I969. Bovine tandem fusion and infertility (Abstr.). Hereditas, 63, 453-454.

HARvey M. J. A., I97I. An autosomal translocation in the Charolais breed of cattle. Vet. Rec., 89, IIO-III.

Herschler M. S., Fechieimer N.S., ig66. Centric fusion of chromosomes in a set of bovine triplets. Cytogenetics, 5, 307-312.

HsU T. C., MEAD R. A., 1969. Mechanisms of chromosomal changes in mammalian speciation. In K. Benrrsch ke. Comparative Mammalian Cytogenetics. An International Conference at Dartmouth Medical School, Hannover, New Hampshire. July 29-August 2, I968. Springer Verlag, Berlin, 8-I5.

Natchigal, M., Sahnazarov N., Graffe L. H., Ionescu-Homoriceanu I., I97o. Chromosomal changes during transformation of fetal bovine kidney cells infected with $\mathrm{SV}_{40}$ virus. Rev. Roum. Inframicrobiol., $7,27-40$.

Popescu C. P., rg7r. Deux cas nouveaux de fusion centrique chez les bovins. Ann. Génét. Sél. anim., 3, $52 \mathrm{I}-526$.

Popescu C. P., I972. Mode de transmission d'une fusion centrique dans la descendance d'un bouc (Capra hircus L.) hétérozygote. Ann. Génét. Sél. anim., 4, 355-361.

Popescu C. P., I973. L'hétérochromatine constitutive dans le caryotype bovin normal et anormal. Ann. Génét., 16, I83-I88.

Rieck G. W., Hohn H., Herzog A., 1968. Familial occurence of centromeric chromosome fusion in cattle. Zuchthygiene, 3, II7-I82.

Rugiati S., Fedrigo M., I967. Aberazione cromosomica riscontrata in un toro accondroplasico di razza Romagnola. L'Ateneo Parmense. Acta Bio-Medica, 38, 3-7.

TAYlor K. M., Hungerford D. A., SNyder R. L., r969. Artiodactyl Mammals : their chromosome cytology in relation to patterns of evolution. In K. BENIRSchke. Comparative Mammalian Cytogenetics. An International Conference at Darmouth Medical School, Hannover, New Hampshire, July 29August 2, 1968. Springer, Berlin. 